\title{
DEVELOPMENT OF C-MEANS CLUSTERING BASED ADAPTIVE FUZZY CONTROLLER FOR A FLAPPING WING MICRO AIR VEHICLE
}

\author{
Md Meftahul Ferdaus ${ }^{1, *}$, Sreenatha G. Anavatti ${ }^{1}$, and Matthew A. Garratt ${ }^{1}$, Mahardhika Pratama ${ }^{2}$ \\ ${ }^{1}$ School of Engineering and Information Technology, \\ University of New South Wales at the Australian Defence Force Academy, \\ Canberra, ACT 2612, Australia \\ ${ }^{2}$ School of Computer Science and Engineering, \\ Nanyang Technological University, \\ Singapore 639798, Singapore \\ *E-mail: m.ferdaus@student.unsw.edu.au
}

Submitted: 5th February 2018; accepted: 18th July 2018

\begin{abstract}
Advanced and accurate modelling of a Flapping Wing Micro Air Vehicle (FW MAV) and its control is one of the recent research topics related to the field of autonomous MAVs. Some desiring features of the FW MAV are quick flight, vertical take-off and landing, hovering, and fast turn, and enhanced manoeuvrability contrasted with similar-sized fixed and rotary wing MAVs. Inspired by the FW MAV's advanced features, a four-wing Natureinspired (NI) FW MAV is modelled and controlled in this work. The Fuzzy C-Means (FCM) clustering algorithm is utilized to construct the data-driven NIFW MAV model. Being model free, it does not depend on the system dynamics and can incorporate various uncertainties like sensor error, wind gust etc. Furthermore, a Takagi-Sugeno (T-S) fuzzy structure based adaptive fuzzy controller is proposed. The proposed adaptive controller can tune its antecedent and consequent parameters using FCM clustering technique. This controller is employed to control the altitude of the NIFW MAV, and compared with a standalone Proportional Integral Derivative (PID) controller, and a Sliding Mode Control (SMC) theory based advanced controller. Parameter adaptation of the proposed controller helps to outperform it static PID counterpart. Performance of our controller is also comparable with its advanced and complex counterpart namely SMC-Fuzzy controller.
\end{abstract}

Keywords: adaptive fuzzy, clustering, flapping wing, micro air vehicle

\section{Introduction}

Nowadays, the application of autonomous systems in thecivil and military sector has increased significantly due to the advancement in control theory and electronics devices. Among various autonomous systems, significant effort is invested in modelling and controlling Micro Air Vehicles
(MAVs), and this is one of the latest research topics in the field of autonomous Unmanned Aerial Vehicles (UAVs). By definition MAVs usually have a maximum dimension of $150 \mathrm{~mm}$, their size can be similar to a small bird, and they have a flight velocity of 10-20 $\mathrm{ms}^{-1}$. Among these MAVs, very recently, nature-inspired flapping wing (NIFW) MAVs are becoming popular among researchers. 
Sharp developments in micro-manufacturing techniques have made NIFW MAVs easily realisable. They are smaller in size and requires comparatively lower power than their fixed wings counterpart. The smaller size also provides them with the capability to perform at lower Reynolds numbers which cannot be obtained from rotary wing UAVs. Furthermore, these NIFW MAVs are able to facilitate a huge range of vital manoeuvres like vertical takeoff and landing, gliding, roll banking, backward and sideways flying, which are not possible for similar sized fixed or rotary wing UAVs. Besides, NIFW MAVs have impressive potential in generating rapid acceleration during manoeuvres. The major benefits and feasibility of utilizing NIFW as MAV are described in [1]. These huge benefits of NIFW MAVs over other fixed and rotary wing UAVs have made them worthy of investigation. The flight dynamics of NIFW MAVs, whether bird inspired or insect-inspired is more complex than their rotary or fixed-wing counterparts, since the flight solely depends on the beating motion of the flapping wings. Therefore, researchers have investigated the flight dynamics of various flapping wing creatures in the last two decades $[2,3,4,5,6,7,8]$. By analysing various features of nature-inspired flapping flight, the emphasis on developing NIFW MAVs is increasing in recent times $[9,10,11]$.

Among different flying insects, dragonflies are one of the oldest with preferred mobility than most other insects as portrayed in $[12,13,14,15]$. A dragonfly has four wings with the ability of quick flight, hovering, and fast moves. Inspired by their desired nature, specialists are attempting to develop Dragonfly liked FW MAV (DLFW MAV) model and trying to enhance their control precision. Linear and non-linear dynamics of a DL MAV was developed in [16] from flight test information. Besides, a sliding mode control theory based adaptive controller was proposed in their work to stabilize the longitudinal dynamics. However, their developed MAV was a fixed wing model. A self-learning wing actuation system around a system of bearings for Dragonfly-inspired MAV (DI-MAV) was fabricated in [17]. They used three solenoids to generate three degrees of freedom motion from a wing, where the solenoids are controlled by a motor driver. The drivers got sinusoidal signals from a workstation desktop computer. A numerical model of a dragonfly robot was developed in [12]. The model was tested with an ordinary PID and fractional order PID control algorithms in a simulation. Their simulated model was able to mimic the kinematics and dynamics of a dragonfly. An insectbased FW MAV was fabricated in [18], which consisted of two fixed and two flapping wings. Their FW MAV can generate adequate vertical thrust to lift-off a weight of 14.76 grams at $10 \mathrm{~Hz}$ frequency. The maximum achievable flapping frequency was $12.4 \mathrm{~Hz}$, which produced an average vertical thrust to lift-off a weight of nearby 24 grams at an applied voltage of $3.7 \mathrm{~V}$. Their FW-MAV exhibited a fruitful free flight with an acceptable control accuracy. A flight controller by utilizing a Linear Quadratic Regulator (LQR) technique was developed in [19] for DLFW MAV. Their DLFW MAV model was linearised to fit with the LQR flight controller. Besides, they have used an iterative learning based tuner to tune the input weighting matrix of LQR to deal with un-modelled parameters. To summarize, up to this point most of the strategies to model and control the FW MAV depend on first principle procedures, the exact numerical model is compulsory to manage their performance. Nevertheless, the FW MAVs are profoundly nonlinear and over-actuated systems. They may contain different vulnerabilities. An exact numerical model of FW MAVs considering these features is challenging to achieve. A smart solution to these issues is the employment of model-free knowledge-based datadriven techniques.

The data-driven modelling and control can play an important role in NIFW MAV system since they don't require any mathematical model. Some of the commonly used non-linear data-driven modelling and control techniques are describing the function method, block structured systems, fuzzy logic, neural networks, and Nonlinear Autoregressive Moving Average Model with Exogenous inputs (NARMAX methods). Among these techniques, fuzzy logic and neural network systems are promising since they demonstrate learning capability from a set of data and approximate reasoning trait of human beings. They can cope with the imprecision and uncertainty of the decision-making process. In recent times fuzzy logic and neural networks are employed to model and control various MAVs [20, 21, 22, 23, 24, 25, 26, 27, 28]. 
A Spiking Neural Network (SNN) to control an FW MAV called RoboBee was proposed in [29]. In [30], a Neural Immunology Network (NIN) based controller was proposed. NIN was inspired by the memory and immune system. Their controller can control the motion of FW MAVs by considering various system non-linearities. Besides, their control method can deal effectively with external perturbations and parameter variations since they do not need any precise dynamics model. A direct adaptive (DA) and hybrid adaptive fuzzy controller (HAFC) was developed in [31] to control dragonflylike FW MAV model by simulation. Better trajectory tracking performance is observed from the HAFC than the DAFC.

Due to the successful implementation and evaluation of various neuro and fuzzy technique in $\mathrm{FW}$ $\mathrm{MAV}$, in this work a FCM clustering based T-S fuzzy system is utilized to identify the FW MAV. In addition, a PD-like adaptive fuzzy controller is developed to control the altitude of the FW MAV.

\section{Fuzzy Modelling and Adaptive Control of Flapping Wing Micro- Air Vehicle}

The FW MAV used in our work is a simulated nature-inspired insect robot with four wings. The development process of the NIFW MAV flight simulator is described in [32]. From this flight simulator the data has been collected to develop the fuzzy based identification and an adaptive controller. Due to the high cost and time consumption to develop and set-up experimental flight test, the utilization of such flight simulators are usual. In this flight simulator, the wing kinematics for a wing flapping in an inclined stroke plane are obtained from the derivation described in [33]. The flapping angle $(\phi)$ in the flapping profile of the FW MAV can be expressed as follows

$$
\phi(t)=\frac{\phi_{f a}}{2} \cos (\pi f t),
$$

where $\phi_{f a}$ is the flapping amplitude in radian, $f$ is the flapping frequency in $\mathrm{Hz}, t$ is the time is second. The angle of attack $(\alpha)$ can be presented as

$$
\alpha=\alpha_{m a}-\alpha_{0} \sin (\omega d t+\psi),
$$

where $\alpha_{m a}$ is mean angle of attack in radian, $\alpha_{0}$ is an amplitude of pitching oscillation in radian, $d t$ is time step in seconds and $\psi$ is the phase difference between the pitching and plunging motion. All the four wings of the FW MAV follows the same flapping profile.

In the simulator, each wing is controlled by an actuator. A symbolic diagram or body coordination of four wing, NIFW MAV is exhibited in Figure 1.

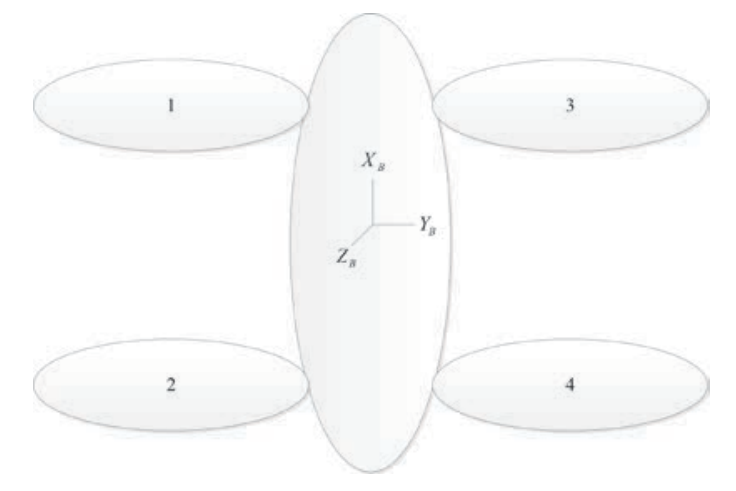

Figure 1. Body coordination of a NIFW MAV.

Numbers indicate the actuator number

Each actuator is controlled by eight (8) flapping parameters namely 1) stroke plane angle (in rad), 2) flapping frequency (in $\mathrm{Hz}$ ), 3) flapping amplitude (in rad), 4) mean angle of attack (inrad), 5) amplitude of pitching oscillation (in rad), 6) phase difference between the pitching and plunging motion, 7) time step (in sec), 8) kappa, set as zero in the plant. A parametric analysis is accomplished to find the dominant flapping parameter. After a complete parametric analysis, it is observed that among eight parameters the flapping amplitude is the dominant one to control the NIFW MAV. Effects of changing the flapping amplitude to some major manoeuvring of a NIFW MAV are summarized in Table 1.

Table 1. Effects of flapping amplitude in different manoeuvring of NIFW MAV

\begin{tabular}{|c|c|c|}
\hline Actuators & $\begin{array}{c}\text { Flapping } \\
\text { amplitude, } \phi_{0} \\
\text { (degree) }\end{array}$ & Action \\
\hline $1,2,3,4$ & 90 & Take-off \\
\hline 1,2 and 3,4 & 90 and 60 & Roll-right \\
\hline 1,2 and 3,4 & 60 and 90 & Roll-left \\
\hline 1,3 and 2,4 & 90 and 60 & Pitch-up \\
\hline 1,3 and 2,4 & 60 and 90 & Pitch-down \\
\hline
\end{tabular}




\subsection{Fuzzy Clustering Based Modelling of the NIFW MAV}

From the NIFW MAV flight simulator, the input-output data is collected to develop the data-driven model, where the four input datasets $\left(u_{1}(t), u_{2}(t), u_{3}(t), u_{4}(t)\right)$ are the four flapping amplitudes applied to four actuators. The three-dimensional (3D) rotational velocities $\left(\omega_{b x}, \omega_{b y}, \omega_{b z}\right)$ and translational velocities $\left(v_{b x}, v_{b y}, v_{b z}\right)$ of the NIFW MAV body are six output datasets. These four inputs $\left(u_{1}(t), u_{2}(t), u_{3}(t), u_{4}(t)\right)$ and delayed outputs $\left(\omega_{b x}(t-1), \omega_{b y}(t-1), \omega_{b z}(t-1), v_{b x}(t-1), v_{b y}(t-\right.$ $\left.1), v_{b z}(t-1)\right)$ are the inputs to the Multi-Input Multi-output (MIMO) nonlinear NIFW MAV model, which can be expressed as follows

$F W(t)=f\left(u_{1}(t), u_{2}(t), u_{3}(t), u_{4}(t), \omega_{b x}(t-1)\right.$, $\left.\omega_{b y}(t-1), \omega_{b z}(t-1), v_{b x}(t-1), v_{b y}(t-1), v_{b z}(t-1)\right)$,

where $F W(t)$ is the MIMO NIFW MAV model. In FCM, a data sample may belong to more than one cluster with a degree of belongingness that varies between 0 to 1 , where the integration of degrees of belongingness of a data sample to all groups is always one as expressed below

$$
\sum_{i=1}^{c} \mu_{i j}=1, \forall j=1, \ldots, n,
$$

where, $i=1,2, \ldots, c ; c$ is the number of clusters, $j=1,2, \ldots, n ; n$ is the number of inputs.

However, the FCM still requires a cost function to be minimized during partitioning the data set. The cost function in FCM can be expressed as follows

$$
J(X ; U, V)=\sum_{i=1}^{c} J_{i}=\sum_{i=1}^{c} \sum_{j=1}^{n} \mu_{i j}^{m} d_{i j}^{2},
$$

where $U=\left[\mu_{1 j}, \mu_{2 j}, \ldots, \mu_{c j}\right]$ is a fuzzy partition matrix of dataset $X$, and $X=\left\{x_{1}, x_{2}, \ldots, x_{n}\right\} ; V=$ $\left[v_{1}, v_{2}, \ldots, v_{c}\right]$ is a vector of cluster centers, $v_{i} \in \mathfrak{R}$; $\mu_{i j}$ ranges between 0 to $1 ; d_{j i}=\left\|x_{j i}-v_{j i}\right\|$ is the Euclidian distance between the $i-t h$ cluster centre and $i-t h$ data point for the $j-t h$ rule; $m \in[1, \infty)$ is a weighting exponent.

The two conditions to reach the minimum for
Equation (5) are as follows

$$
\begin{gathered}
y_{i}=\frac{\sum_{j=1}^{n} \mu_{i j}^{m} x_{j}}{\sum_{j=1}^{n} \mu_{i j}^{m}}, \\
\mu_{i j}=\frac{1}{\sum_{k=1}^{c}\left(\frac{d_{i j}}{d_{k j}}\right)^{2 /(m-1)}} .
\end{gathered}
$$

The FCM algorithm repeatedly performs through Equation (6) and (7) until no more improvement is observed. The efficiency of Equation (6) and (7) and convergence of the FCM algorithm is proven in [34].

In batch training operation, the algorithm of FCM based T-S fuzzy model to determine the cluster centres $v_{i}$ and the membership matrix $U$ is presented in Algorithm below.

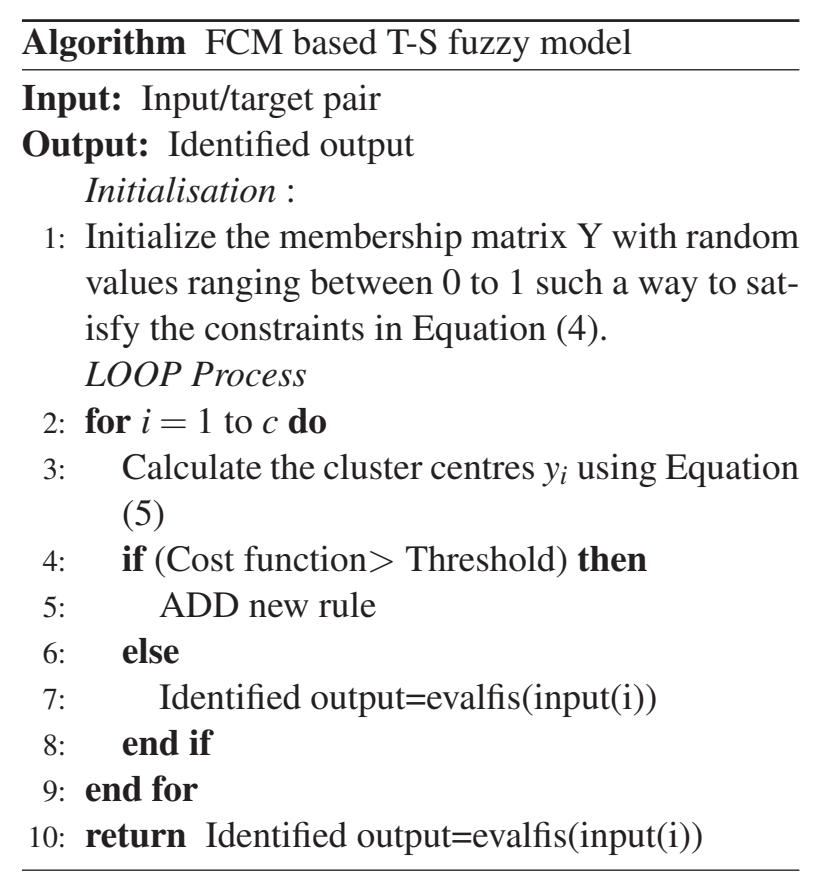

In FCM, the performance has a dependency on the initial membership matrix values, which suggest running the algorithm for a few times.

\subsection{Development of Adaptive Fuzzy Con- troller}

A T-S fuzzy structure has been utilized to develop the adaptive fuzzy controller in this work. The FCM clustering technique is utilized to adapt the premise parameters such as centres and widths of the membership function of the controller. It has 
also been utilized to adapt the consequent part of T-S fuzzy system of the adaptive controller. The proposed controller is trained with a stable PD controller, which has been exposed in Figure 2. However, the fuzzy controller performs better than the static PD controller since the fuzzy controller can adapt its parameter using FCM technique. The closed-loop adaptive control system is exhibited in Figure 3. The difference between the reference signal and plants output i.e. the error $(e(t))$ is one of the inputs to the controller, and the rate of change of that error $(d e(t) / d t)$ is another input to the controller, which is presented in the input layer of Figure 3. These crisp inputs are being fuzzified in the fuzzification layer, where Gaussian membership functions are utilized. To get the desired signal from the FW MAV model, the fuzzy controller alters the Gaussian membership functions width and centres by utilizing the FCM clustering technique, where the error signal $e(t)$ is utilized as a cost function for the FCM clustering. After this, the 'AND' operation i.e. the product of all membership functions are obtained. Finally, output of the adaptive fuzzy controller is calculated in the output layer as follows

$$
y_{i}=\frac{\sum_{i=1}^{N} w_{i} z_{i}}{\sum_{i=1}^{N} w_{i}}=\Psi . \Theta,
$$

where $i=1,2, \ldots, N ; N$ is the number of rules, $\Psi$ is the product of normalized firing strength and input vector, and $\Theta$ is the vector of consequent parameter, $w_{i}$ is the rule firing strength of the $i-t h$ rule and can be expressed as

$$
w_{i}=\prod_{j=1}^{n} \mu_{A_{j}}^{i},
$$

where $j=1,2, \ldots, n ; n$ is the number of inputs, $\mu_{A_{j}}^{i}$ is the membership function of the $i-t h$ rule and $j-t h$ input. In this work, Gaussian membership function is employed and can be expressed as:

$$
\mu_{A_{j}}^{i}=\exp \left(-\frac{1}{2}\left(\frac{x_{j}-v_{j}^{i}}{\sigma_{j}^{i}}\right)^{2}\right),
$$

where $v_{j}^{i}$ is the center and $\sigma_{j}^{i}$ is the width of the Gaussian membership function for the $i-t h$ rule and $j-t h$ input. In Figure 3, the $z_{i}=a_{0 j}+a_{1 i} x_{1}+$ $a_{2 i} x_{2}+\ldots+a_{n i} x_{n}$ is expressing the consequent part of the $i-t h$ rule, where $a_{0}, a_{1}, a_{2}, \ldots, a_{n}$ are consequent parameters of that rule. In this work, the inputs are $x_{1}=e$ and $x_{2}=\dot{e}$. The controller's output signal goes to the identified NIFW MAV model. Then the model's output is integrated to get the vertical altitude from velocity, and compared with the reference position. The controller tunes its parameter until the model output follows the reference signal, and consequently, the error signal $(e(t))$ becomes zero. In this FCM based adaptive fuzzy controller, five Gaussian membership functions are utilized. Vectors of Initial centers for those membership functions are [-7.386 -4.93 -1.443 2.531 6.483] and $\left[\begin{array}{lllll}-38.24 & -26.62 & -8.747 & 13.63 & 36.7\end{array}\right]$ respectively for the error $e$ and a derivative of error $\dot{e}$, whereas the vectors of widths are [5.837 5.055 5.667 6.274

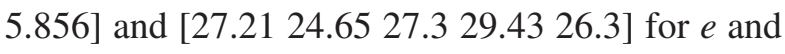
$\dot{e}$ respectively.

\section{Stability Analysis of the Adaptive Fuzzy Con- troller:}

Usually, the stability test requires a mathematical model of the plant to be controlled. However, attaining a proper mathematical model of a highly non-linear over-actuated system like NIFW MAV is too difficult. In such situation, the model free adaptive fuzzy controller is an appropriate solution. In this work, the closed-loop stability of the adaptive fuzzy controller is ensured with the assistance of the PD controller as explained and proved in $[27,35]$.

Theorem 1 The adaptation laws for the proposed adaptive fuzzy controller are expressed as

$$
\begin{gathered}
\dot{\Theta}(t)=-k_{p} H(t) \Psi(t) s_{l}(t), \\
\text { where } \Theta(0)=\Theta_{0} \in \Re^{n R \times 1},
\end{gathered}
$$

where $n$ is the number of inputs, $R$ is the number of rules, $\Theta_{0}$ is the initial value of $\Theta$. The term $H(t)$ of Equation (11) can be updated recursively as follows

$$
\dot{H}(t)=-H(t) \Psi(t) \Psi^{T}(t) H(t),
$$

where $H(0)=H_{0} \in \Re^{n R \times n R}, H_{0}$ is the initial value of $H . n$ is presenting the number of inputs to the controller, and $R$ is the number of rules. These adaptation laws assure a stable closed-loop control system. 


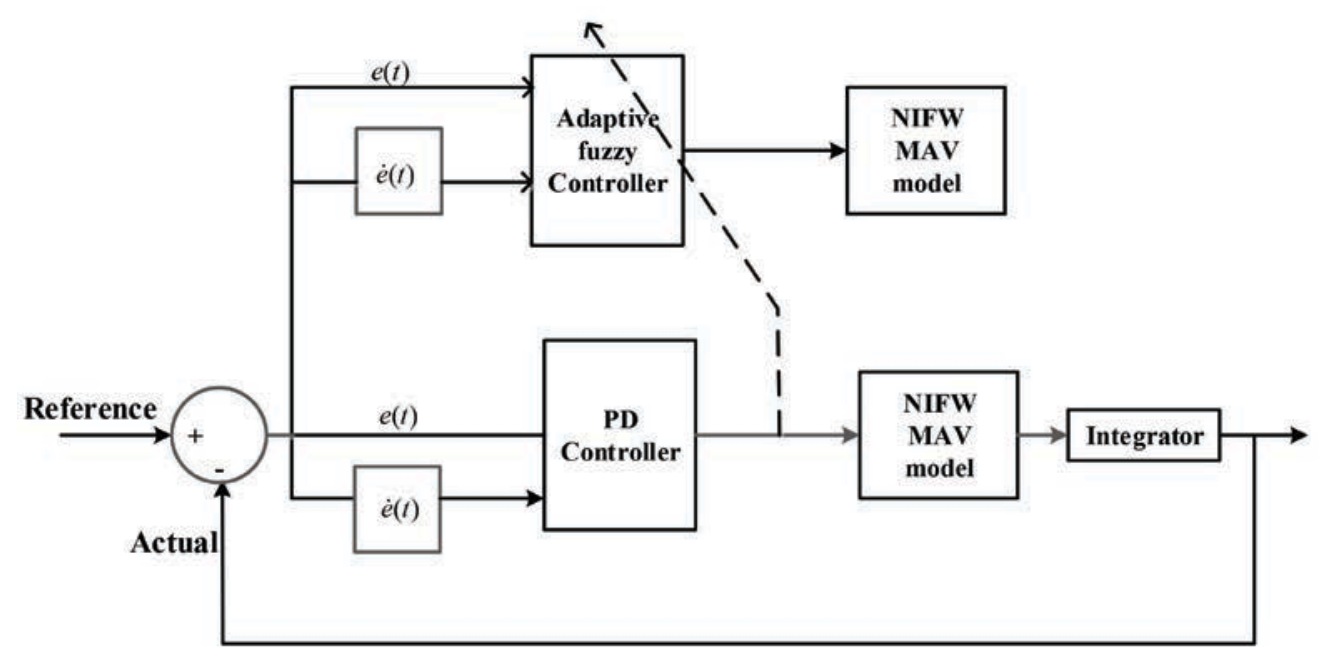

Figure 2. Training of FCM based fuzzy adaptive control system from a stable PD controller

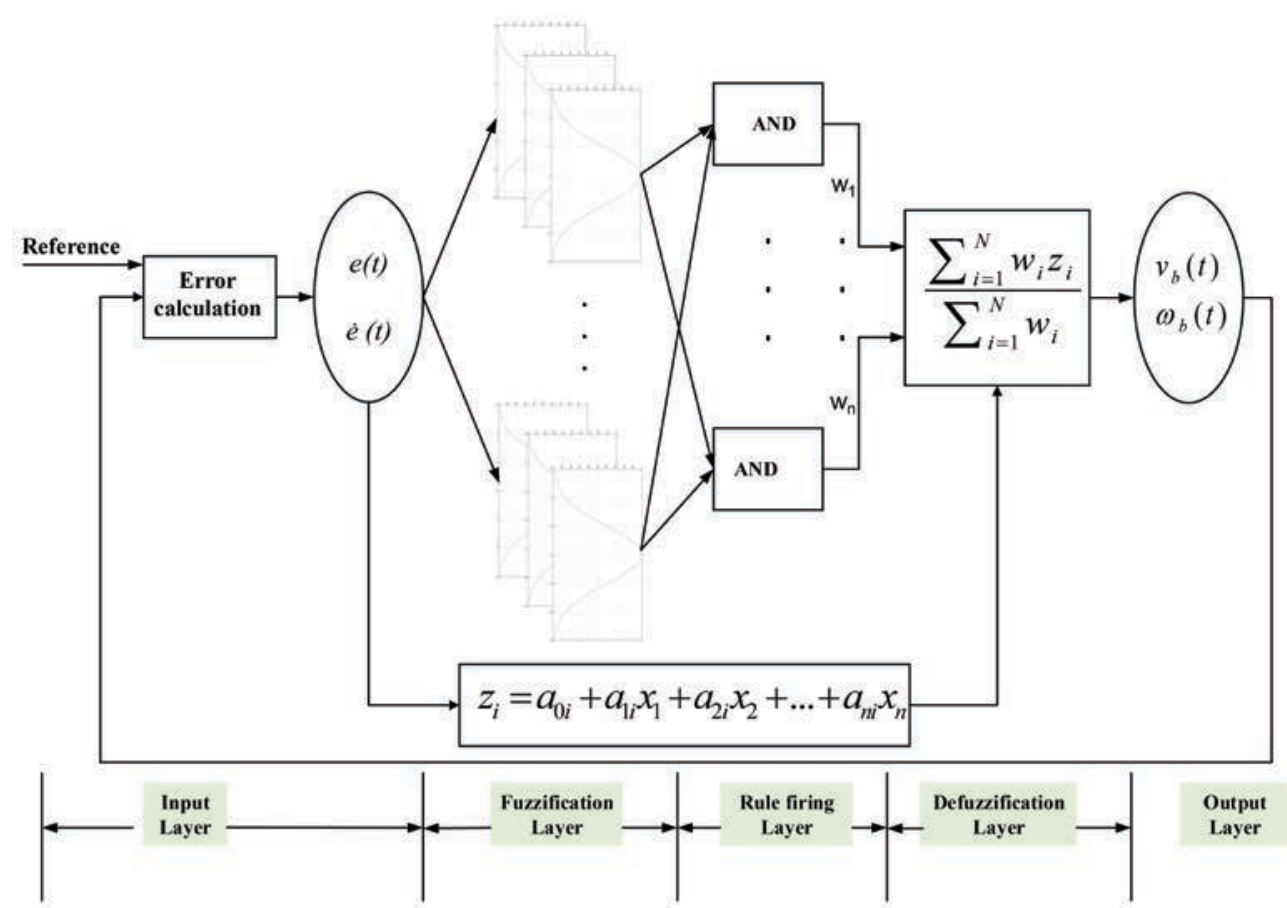

Figure 3. Closed-loop block diagram of FCM based fuzzy adaptive control system 


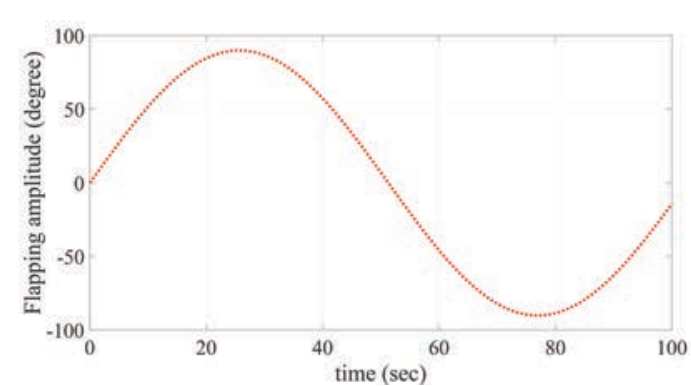

Figure 4. Input: Flapping amplitude

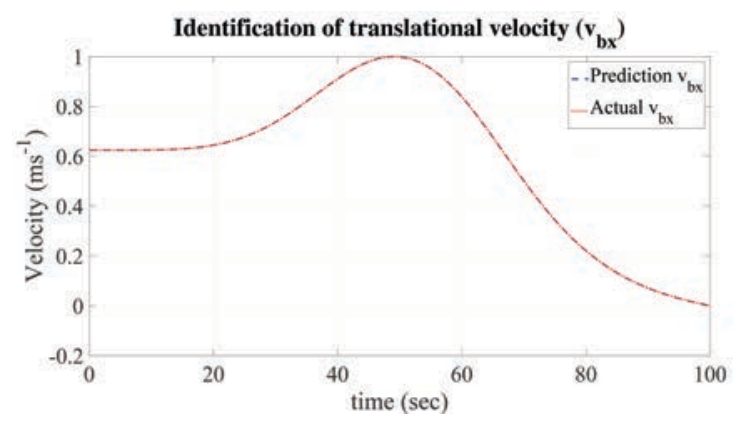

(a) Translational velocity $\left(v_{b x}\right)$

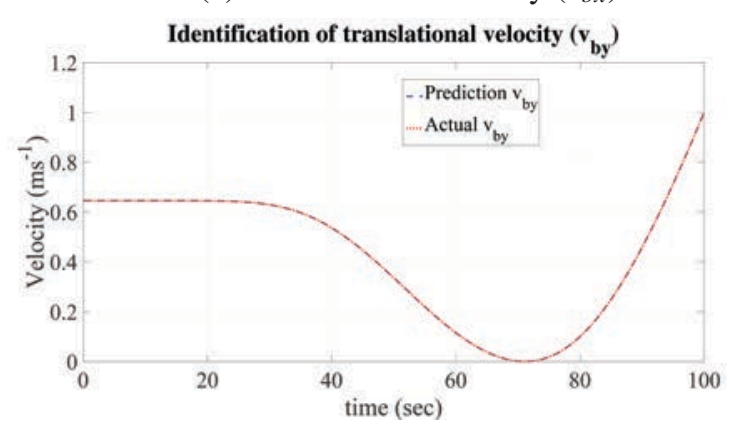

(b) Translational velocity $\left(v_{b y}\right)$

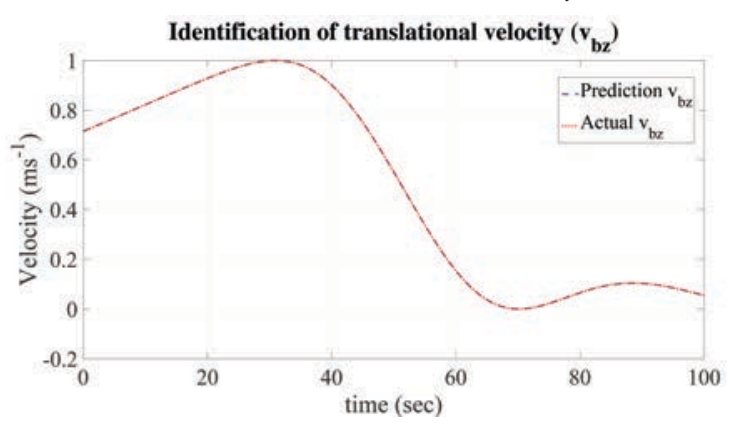

(c) Translational velocity $\left(v_{b z}\right)$

Figure 5. Identification of FW MAVs translational velocity $\left(v_{b x}, v_{b y}, v_{b z}\right)$

\section{Results and Discussion}

The data used for the MIMO nonlinear NIFW MAV system identification is based on a $100 \mathrm{sec}-$ onds simulation in Simulink with a time step of
0.01 seconds. In a physical NIFW MAV model, it can change its flapping amplitude within a certain range, which is between $-90^{\circ}$ and $90^{\circ}$. Therefore, a sinusoidal flapping amplitude varying between $-90^{\circ}$ and $90^{\circ}$ is applied to each actuator of all four wings of the NIFW MAV as shown in Figure 4, which helps the FCM clustering based NIFW MAV model to get the input datasets within the maximum range. In this technique, T-S fuzzy model with three (3) Gaussian membership function is utilized. From Figure 5 and Figure 6 it is clearly observed that all the translation velocities $\left(v_{b x}, v_{b y}\right.$, and $\left.v_{b z}\right)$, and the rotational velocities $\left(\omega_{b x}, \omega_{b y}\right.$, and $\left.\omega_{b z}\right)$ are identified with a negligible error.

The adaptive T-S fuzzy controller's performance is evaluated with respect to various reference signals and the performance is compared with a PID controller, and SMC theory based fuzzy controller developed in [36]. In this work, the considered trajectories for tracking altitude are as follows: 1) constant height of $10 \mathrm{~m}$; 2) sinusoidal wave function with an amplitude of $1 \mathrm{~m}$ and frequency of 1 $\mathrm{Hz} ; 3)$ square wave function with an amplitude of $1 \mathrm{~m}$ and frequency of $0.1 \mathrm{~Hz}$; and 4) different step functions. In all the figures, the proposed controller is named as "FCM-TS-Fuzzy", whereas the benchmarked controllers are named as "SMC-Fuzzy", and "PID". At first, tracking performance of the controllers are observed for a trajectory of constant height. The results are observed in Figure 7, from where it is observed that the proposed controller performs better than the PID controller. The performance is also comparable with SMC-Fuzzy controller. Besides, comparatively higher overshoot is observed from the PID controller at the transient state. After that, the performance is witnessed for a sinusoidal reference signal, where an improved performance is observed from the FCM-TS-Fuzzy controller as exposed in Figure 8. A square wave pulse signal is also inserted into the closed loop system to observe the efficacy of the proposed adaptive fuzzy controller. Our proposed controller outperforms the PID and SMC-Fuzzy controller as shown in Figure 9. Finally, three different types of step functions such as $Z_{b_{d}}(t)=10 u(t-20), Z_{b_{d}}(t)=$ $5 u(t)+5 u(t-20), Z_{b_{d}}(t)=-5 u(t)+10 u(t-20)$ are used as reference signal to check the proposed controller's performance. Here $u(t)$ is a unit step function. Satisfactory and better performance than the PID controller is recorded in all cases as shown 
in Figure 10, Figure 11, and Figure 12. The SMCFuzzy controller performs slightly better than the proposed controller. However, the results are comparable with a simple structure of the proposed controller compare to the SMC-Fuzzy controller. Besides, the root mean square error (RMSE) for PID, SMC-Fuzzy and the proposed adaptive fuzzy controller in all cases are tabulated in Table 1, where RMSEs of the fuzzy controller are less than the PID controller, and very close to the SMC-Fuzzy controller.

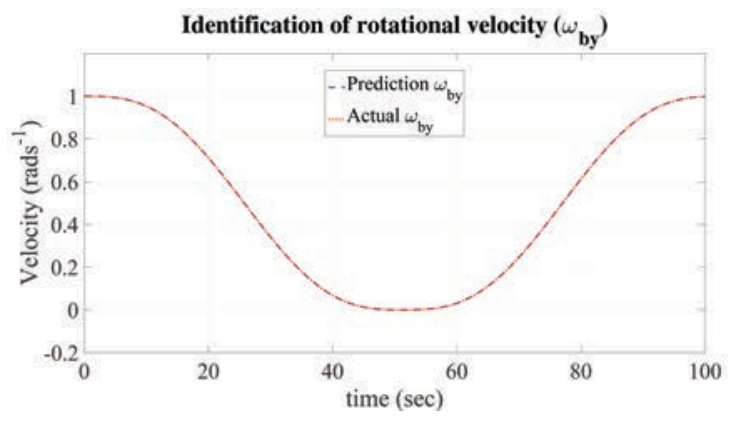

(a) Rotational velocity $\left(\omega_{\text {by }}\right)$

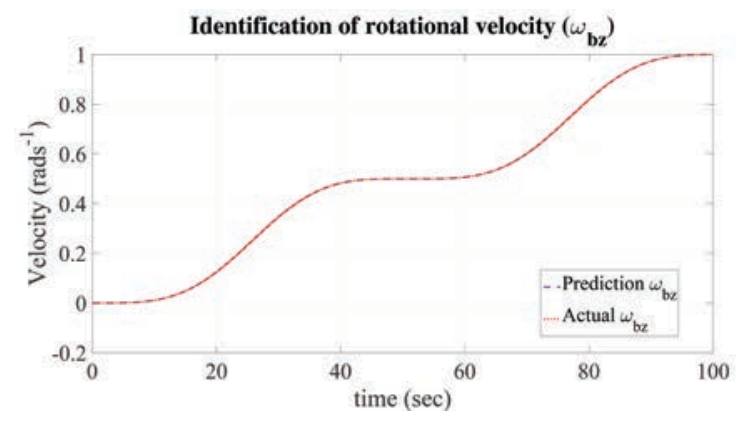

(b) Rotational velocity $\left(\omega_{b z}\right)$

Figure 6. Identification of FW MAVs rotational velocity $\left(\omega_{b y}, \omega_{b z}\right)$

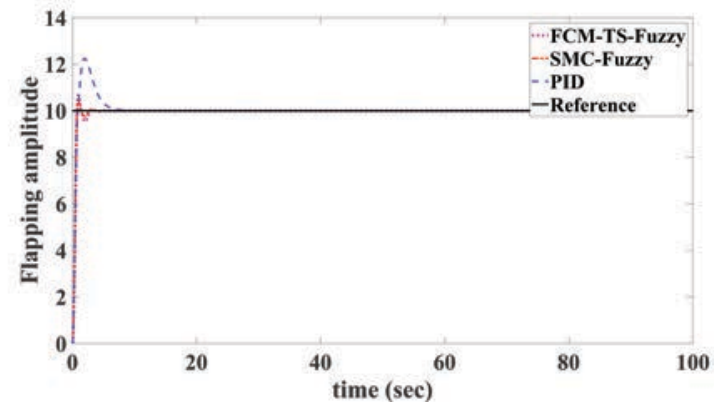

Figure 7. Altitude tracking performance of NIFW MAV controllers for a constant height trajectory

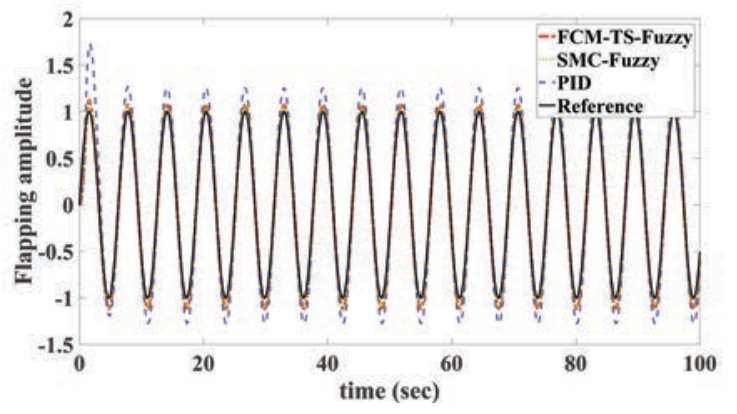

Figure 8. Altitude tracking performance of NIFW MAV controllers for a sinusoidal trajectory

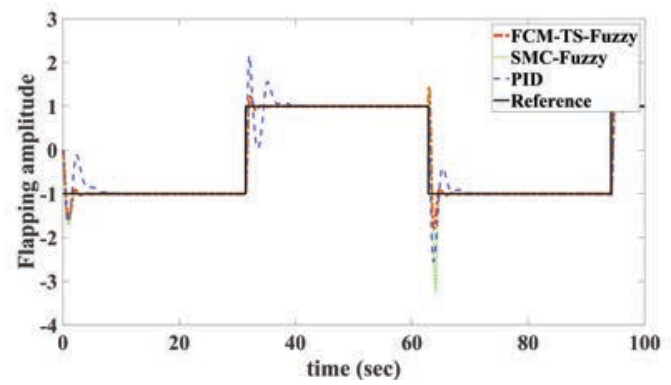

Figure 9. Altitude tracking performance of NIFW MAV controllers for a square wave trajectory

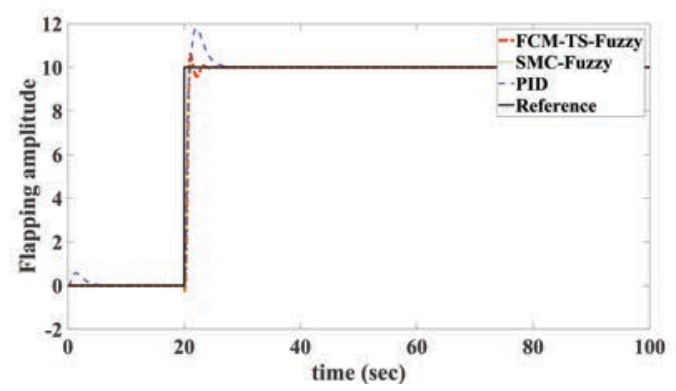

Figure 10. Altitude tracking performance of NIFW MAV controllers for a step wave trajectory changing amplitude from $0 \mathrm{~m}$ to $10 \mathrm{~m}$ after $20 \mathrm{sec}$.

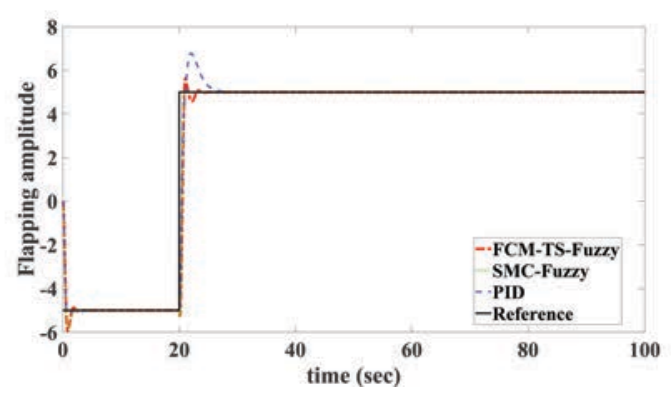

Figure 11. Altitude tracking performance of FW MAV controllers for a step wave trajectory changing amplitude from $-5 \mathrm{~m}$ to $5 \mathrm{~m}$ after $20 \mathrm{sec}$. 


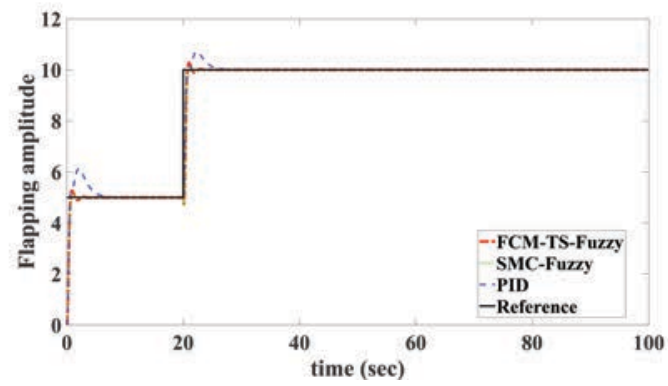

Figure 12. Altitude tracking performance of NIFW MAV controllers for a step wave trajectory changing amplitude from $5 \mathrm{~m}$ to $10 \mathrm{~m}$ after $20 \mathrm{sec}$.

Table 2. Controllers performance (Measured RMSE)

\begin{tabular}{|l|c|c|c|}
\hline \multirow{2}{*}{$\begin{array}{l}\text { Reference } \\
\text { Signal }\end{array}$} & $\begin{array}{c}|c| \\
\text { FCM- } \\
\text { TS- } \\
\text { Fuzzy }\end{array}$ & $\begin{array}{l}\text { SMC- } \\
\text { Fuzzy }\end{array}$ & PID \\
\hline $\begin{array}{l}\text { Constant } \\
\text { height }\end{array}$ & 0.5693 & 0.5876 & 0.6630 \\
\hline Sinusoidal & 0.0737 & 0.0790 & 0.2096 \\
\hline Square wave & 0.2039 & 0.2098 & 0.2493 \\
\hline Step 1 & 0.4023 & 0.3856 & 0.4000 \\
\hline Step 2 & 0.6266 & 0.5678 & 0.6701 \\
\hline Step 3 & 0.6888 & 0.6695 & 0.7188 \\
\hline
\end{tabular}

\section{Conclusion}

Acquiring an exact numerical model and the control of a highly nonlinear over-actuated complex system like NIFW MAV is difficult. Besides, the uncertainties are hard or sometimes impossible to incorporate in such a model. Propelled by various points of interest of model-free techniques using neural networks, and fuzzy logic systems, in this work an FCM clustering based nonlinear fuzzy MIMO NIFW MAV model is developed, where the datasets are recorded from a built-up NIFW MAV flight simulator. Moreover, an adaptive fuzzy controller is developed and employed to control the MAVs altitude. In the developed controller, the FCM clustering is used to tune the antecedent parameters, whereas the PD theory is utilized to adapt the consequent parameters. To evaluate the controller's performance, it is contrasted with a PID controller with respect to constant height, sinusoidal wave, square wave, and three different step functions. In all cases, our developed adaptive fuzzy controller outperforms the PID controller. In compare to the SMC-Fuzzy controller, the architecture of the proposed fuzzy controller is much simpler. However, a comparable performance is witnessed from the proposed controller. The proposed fuzzy controller helps the MAV to follow various desired trajectories with RMSE of only 0.5693 , $0.0737,0.2039,0.4023,0.6266$ and 0.7188 . In future, our adaptive fuzzy controller will be advanced to an evolving controller by utilizing learning machine algorithms and will be implemented in a NIFW MAV hardware.

\section{References}

[1] C. P. Ellington, The novel aerodynamics of insect flight: applications to micro-air vehicles, Journal of Experimental Biology, vol. 202, no. 23, pp. 34393448, 1999.

[2] W. Shyy, Y. Lian, J. Tang, D. Viieru, and H. Liu, Aerodynamics of low Reynolds number flyers. Cambridge University Press, 2007, vol. 22.

[3] W. Shyy, H. Aono, S. K. Chimakurthi, P. Trizila, C.K. Kang, C. E. Cesnik, and H. Liu, Recent progress in flapping wing aerodynamics and aeroelasticity," Progress in Aerospace Sciences, vol. 46, no. 7, pp. 284-327, 2010.

[4] H. Tennekes, The simple science of flight: from insects to jumbo jets. MIT press, 2009.

[5] A. P. Willmott and C. P. Ellington, The mechanics of flight in the hawkmoth manduca sexta. i. kinematics of hovering and forward flight. Journal of Experimental Biology, vol. 200, no. 21, pp. 2705-2722, 1997.

[6] S. P. Sane, The aerodynamics of insect flight, Journal of experimental biology, vol. 206, no. 23, pp. 4191-4208, 2003.

[7] Y. Lin, Y. Xu, H. Chen, M. J. Bender, A. L. Abbott, and R. Müller, Optimal Threshold and LoG Based Feature Identification and Tracking of Bat Flapping Flight, in Applications of Computer Vision (WACV), 2017 IEEE Winter Conference on. IEEE, 2017, pp. 418-426.

[8] S. M. Nogar, A. Gogulapati, J. J. McNamara, A. Serrani, M. W. Oppenheimer, and D. B. Doman, Control-Oriented Modeling of Coupled Electromechanical-Aeroelastic Dynamics for Flapping-Wing Vehicles," Journal of Guidance, Control, and Dynamics, 2017.

[9] J. Zhang and X. Deng, Resonance principle for the design of flapping wing micro air vehicles, IEEE 
Transactions on Robotics, vol. 33, no. 1, pp. 183197, 2017.

[10] C. Zhang and C. Rossi, A review of compliant transmission mechanisms for bio-inspired flappingwing micro air vehicles, Bioinspiration \& biomimetics, vol. 12, no. 2, p. 025005, 2017.

[11] M. W. Oppenheimer, D. O. Sigthorsson, I. E. Weintraub, and D. B. Doman, Wing Design and Testing for a Tailless Flapping Wing Micro Air Vehicle, in AIAA Guidance, Navigation, and Control Conference, 2017, p. 1271.

[12] M. S. Couceiro, N. Ferreira, and J. Machado, Modeling and control of a dragonfly-like robot, Journal of Control Science and Engineering, vol. 2010, p. 5, 2010.

[13] J. Sun, C. Pan, J. Tong, and J. Zhang, Coupled model analysis of the structure and nano-mechanical properties of dragonfly wings, IET nanobiotechnology, vol. 4, no. 1, pp. 10-18, 2010.

[14] M. Okamoto, K. Yasuda, and A. Azuma, Aerodynamic characteristics of the wings and body of a dragonfly, Journal of Experimental Biology, vol. 199, no. 2, pp. 281-294, 1996.

[15] S. Sudo, K. Tsuyuki, T. Ikohagi, F. Ohta, S. Shida, and J. Tani, A study on the wing structure and flapping behavior of a dragonfly, JSME International Journal Series C Mechanical Systems, Machine Elements and Manufacturing, vol. 42, no. 3, pp. 721729, 1999.

[16] J. S. Jang and C. Tomlin, Longitudinal stability augmentation system design for the DragonFly UAV using a single GPS receiver, in AIAA Guidance, Navigation, and Control Conference, AIAA, vol. 5592, 2003

[17] J. M. Kok and J. Chahl, Design and manufacture of a self-learning flapping wing-actuation system for a dragonfly-inspired MAV, in 54th AIAA Aerospace Sciences Meeting, 2016, p. 1744.

[18] Q.-V. Nguyen, W. L. Chan, and M. Debiasi, Design, fabrication, and performance test of a hovering-based flapping-wing micro air vehicle capable of sustained and controlled flight, 2014.

[19] C.-p. Du, J.-x. Xu, and Y. Zheng, Application of iterative learning tuning to a dragonfly-like flapping wing micro aerial vehicle, in Control and Decision Conference (CCDC), 2016 Chinese. IEEE, 2016, pp. 4215-4220.

[20] M. M. Ferdaus, S. G. Anavatti, M. Pratama, and M. A. Garratt, Online Identification of a Rotary Wing Unmanned Aerial Vehicle from Data Streams," 2017.
[21] S. B. Hu, W. H. Lu, Z. Y. Chen, L. Lei, and Y. X. Zhang, Attitude control of flapping wing micro aerial vehicle based on double fuzzy sliding mode control, in Advanced Materials Research, vol. 468. Trans Tech Publ, 2012, pp. 704-707.

[22] A. A. Al-Mahasneh, S. G. Anavatti, and M. Garratt, Nonlinear Multi-Input Multi-Output System Identification using Neuro-Evolutionary Methods for a Quadcopter, IEEE, pp. 217-222, 2017.

[23] M. M. Ferdaus, S. G. Anavatti, M. A. Garratt, and M. Pratama, Fuzzy Clustering based Nonlinear System Identification and Controller Development of Pixhawk based Quadcopter, in Advanced Computational Intelligence (ICACI), 2017 IEEE International Conference on. IEEE, 2017, pp. 223-230.

[24] M. M. Ferdaus, S. G. Anavatti, M. A. Garratt, and M. Pratama, Fuzzy Clustering based Modelling and Adaptive Controlling of a Flapping Wing Micro Air Vehicle, in Computational Intelligence (IEEE SSCI), 2017 IEEE Symposium Series on. IEEE, 2017, pp. 1914-1919.

[25] M. M. Ferdaus, M. Pratama, S. G. Anavatti, and M. A. Garratt, Evolving Neuro-Fuzzy System based Online Identification of a Bio-inspired Flapping Wing Micro Aerial Vehicle, in Computational Intelligence (IEEE SSCI), 2017 IEEE Symposium Series on. IEEE, 2017, pp. 2840-2847.

[26] C. Zhang, Design and control of flapping wing micro air vehicles, 2016. [Online]. Available: http://oa.upm.es/44319/

[27] M. Ferdaus, M. Pratama, S. G. Anavatti, M. A. Garratt, and Y. Pan, Generic evolving self-organizing neuro-fuzzy control of bioinspired unmanned aerial vehicles, arXiv preprint arXiv:1802.00635, 2018.

[28] A. J. Al-Mahasneh, S. Anavatti, and M. Garratt, Altitude identification and intelligent control of a flapping wing micro aerial vehicle using modified generalized regression neural networks, in Computational Intelligence (IEEE SSCI), 2017 IEEE Symposium Series on. IEEE, 2017.

[29] T. S. Clawson, S. Ferrari, S. B. Fuller, and R. J. Wood, Spiking neural network (SNN) control of a flapping insect-scale robot, in Decision and Control (CDC), 2016 IEEE 55th Conference on. IEEE, 2016, pp. 3381-3388.

[30] L. Weng, M. Xia, K. Hu, and A. Wang, Micro Aerial Vehicle (MAV) Flapping Motion Control Using an Immune Network with Different Immune Factors," International Journal of Advanced Robotic Systems, vol. 10, no. 8, p. 305, 2013. 
[31] M. S. Couceiro, N. M. Ferreira, and J. T. Machado, Hybrid adaptive control of a dragonfly model, Communications in Nonlinear Science and Numerical Simulation, vol. 17, no. 2, pp. 893-903, 2012.

[32] J. Kok and J. Chahl, A low-cost simulation platform for flapping wing MAVs, in SPIE Smart Structures and Materials+ Nondestructive Evaluation and Health Monitoring. International Society for Optics and Photonics, 2015, pp. 94 290L-94 290L.

[33] Z. J. Wang, The role of drag in insect hovering, Journal of Experimental Biology, vol. 207, no. 23, pp. 4147-4155, 2004.

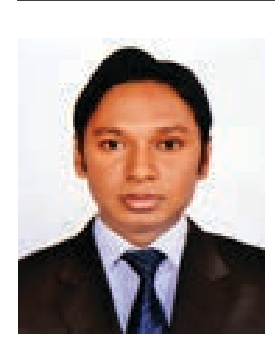

Md Meftahul Ferdaus completed his BSc. in Electrical and Electronic Engineering from RUET Bangladesh in 2011 and then done M.Sc. in Mechatronics Engineering from IIUM Malaysia in 2015, where he achieved the best student award in Masters programme in faculty of engineering. Now he is a Ph.D. Candidate at the University of New South Wales. He is also a visiting PhD scholar at the Nanyang Technological University Singapore from October 2017. His research Interest includes Machine Learning, Evolving Neuro-fuzzy systems, intelligent control system, UAVs, MAVs, MR materials, etc. He has published several papers in peer reviewed high-quality journals and conferences. Besides, he is working as a reviewer in several reputed journals like IEEE Transaction on Industrial Electronics, Journal of Intelligent and Fuzzy Systems, International Journal of Control Automation and Systems, Journal of Mechanical Science and Technology etc.

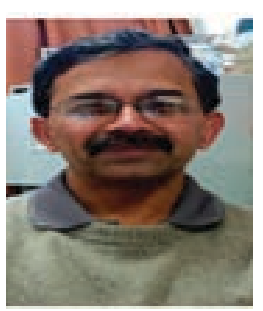

Sreenatha Anavatti is a senior lecturer with SEIT, UNSW Canberra, Australia. Before moving to Australia in 1998, he was with Indian Institute of Technology Bombay, Mumbai, as an Associate Professor. His research interests involve the application of artificial intelligence techniques like Fuzzy and Neural systems for UAVs, Underwater Vehicles and Ground Vehicles. The research interests include Identification of dynamic systems, Control of dynamic systems, Navigation and Path planning for autonomous vehicles, Evolutionary Fuzzy systems and their applications to dynamic systems. He has supervised several Ph.D. scholars over the years.

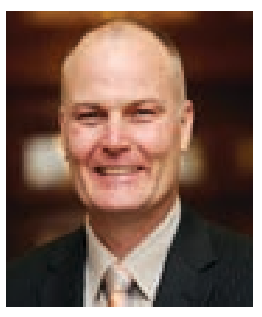

Matthew A. Garratt received the Ph.D. degree in robotics from Australian National University (ANU), Canberra, ACT, Australia, in 2007. He was an Aeronautical Engineer with the Royal Australian Navy, Canberra, before working for two years in the field of computer-aided engineering tools.
[34] J. C. Bezdek, A convergence theorem for the fuzzy isodata clustering algorithms, IEEE transactions on pattern analysis and machine intelligence, no. 1, pp. $1-8,1980$.

[35] M. M. Ferdaus, M. Pratama, S. G. Anavatti, and M. A. Garratt, A generic self-evolving neurofuzzy controller based high-performance hexacopter altitude control system, arXiv preprint arXiv:1805.02508, 2018.

[36] M. M. Ferdaus, S. G. Anavatti, M. A. Garratt, and M. Pratama, Development of a sliding mode control based adaptive fuzzy controller for a flapping flight, arXiv preprint arXiv:1806.02945, 2018.

In 1999, he joined ANU, where he helped build and test an autonomous helicopter using biologically inspired vision for a Defense Advanced Research Projects Agency (DARPA) funded project. Since 2001, he has been with the University of New South Wales, Canberra, where he is currently an Associate Professor. His main research areas are sensing and control for autonomous systems

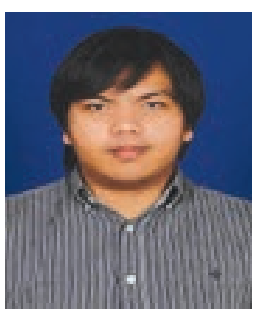

Mahardhika Pratama received his $\mathrm{PhD}$ degree from the University of New South Wales, Australia in 2014. He completed his $\mathrm{PhD}$ in 2.5 years with a special approval of the UNSW higher degree committee due to his outstanding $\mathrm{PhD}$ research achievement. He is currently assistant professor at School of Computer Science and Engineering, Nanyang Technological University. Before joining NTU. He worked as a lecturer at the Department of Computer Science and IT, La Trobe University from 2015 till 2017. Prior to joining La Trobe University, he was with the Centre of Quantum Computation and Intelligent System, University of Technology, Sydney as a postdoctoral research fellow of Australian Research Council Discovery Project. Dr. Pratama received various competitive research awards in the past 5 years, namely the Institution of Engineers, Singapore (IES) Prestigious Engineering Achievement Award in 2011, the UNSW high impact publication award in 2013 and 2014. $\mathrm{He}$ recently has been appointed as Indonesias government world-class professor. Dr. Pratama has produced over 64 high-quality papers in journals and conferences and edited one book, and has been invited to deliver keynote speeches in international conferences. Dr. Pratama has led five special sessions and two special issues in prestigious conferences and journals. He currently serves as an editor in chief of International Journal of Business Intelligence and Data Mining and a consultant at Life bytes, Australia. Dr. Pratama is a member of IEEE, IEEE Computational Intelligent Society (CIS) and IEEE System, Man and Cybernetic Society (SMCS), and Indonesian Soft Computing Society (ISC-INA). His research interests involve machine learning, computational intelligent, evolutionary computation, fuzzy logic, neural network and evolving adaptive systems. 